\title{
Role of the dorsal cochlear nucleus in the sound localization behavior of cats
}

\author{
Bradford J. May * \\ Department of Otolaryngology-HNS, The Johns Hopkins University, 505 Traylor Research Bldg., 720 Rutland Avenue, Baltimore, \\ MD 21205-2195, USA \\ Received 7 March 2000; accepted 5 June 2000
}

\begin{abstract}
The role of the dorsal cochlear nucleus (DCN) in directional hearing was evaluated by measuring sound localization behaviors before and after cats received lesions of the dorsal and intermediate acoustic striae (DAS/IAS). These lesions are presumed to disrupt spectral processing in the DCN without affecting binaural time and level difference cues that exit the cochlear nucleus via the ventral acoustic stria. Prior to DAS/IAS lesions, cats made accurate head orientation responses toward sound sources in the frontal sound field. After a unilateral DAS/IAS lesion, subjects showed increased errors in the azimuth and elevation of their responses; in addition, the final orientation of head movements tended to be more variable. Largest deficits in response elevation were observed in the hemifield that was ipsilateral to the lesion. When a second lesion was placed in the opposite DAS/IAS, increased orientation errors were observed throughout the frontal field. Nonetheless, bilaterally lesioned cats showed normal discrimination of changes in sound source location when tested with a spatial acuity task. These findings support previous interpretations that the DCN contributes to sound orientation behavior, and further suggest that the identification of absolute sound source locations and the discrimination between spatial locations involve independent auditory processing mechanisms. (C) 2000 Elsevier Science B.V. All rights reserved.
\end{abstract}

Key words: Dorsal cochlear nucleus; Sound localization; Orientation behavior; Spatial acuity

\section{Introduction}

Cats accurately orient toward randomly placed sound sources in the frontal auditory field (May and Huang, 1996). This biologically relevant behavior is aided by directional cues that are derived from filtering effects of the head-related transfer function (HRTF: the function relating the free-field spectrum of a broadband sound to energy propagating to the eardrum). Rice et al. (1992) have shown that a particularly robust filtering effect of the cat's HRTF is the introduction of a midfrequency spectral notch that changes in frequency with sound source azimuth and elevation (Fig. 1A,B). Cats show well directed responses to sound sources if mid-

\footnotetext{
* Tel.: +1 (410) 955-3162; Fax: +1 (410) 955-1299; E-mail: bmay@bme.jhu.edu
}

frequency localization cues are present in acoustic stimuli (Huang and May, 1996a). For example, the orientation responses of cat M91 fell close to actual sound source locations when stimuli were bursts of broadband noise (Fig. 1C) or bandpass noise with energy at 5-20 $\mathrm{kHz}$ (Fig. 1D). Orientation responses became less accurate and more variable when mid-frequency cues were removed by high-pass filtering the stimuli at $18 \mathrm{kHz}$ (Fig. 1E).

Electrophysiological studies suggest that neural responses in the dorsal cochlear nucleus (DCN) are sensitive to the spectral cues produced by directional filtering properties of the HRTF. This specialization can be seen in the tonotopic organization of the nucleus as an $8-30-\mathrm{kHz}$ bias in the distribution of single-unit best frequencies (Spirou et al., 1993). In addition, the discharge rates of DCN principal cells are modified by the frequency of notched-noise stimuli that mimic HRTFbased localization cues (Spirou and Young, 1991). 

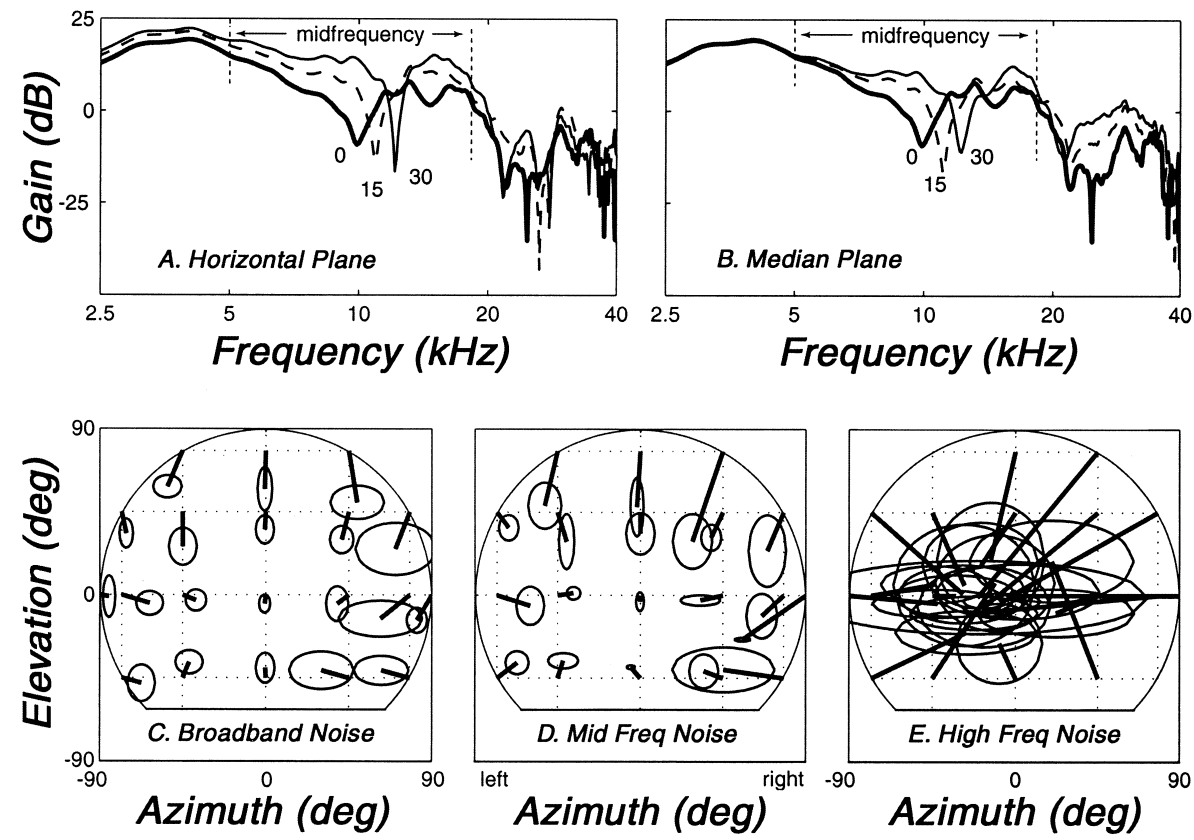

Fig. 1. HRTFs of the cat at different locations in the horizontal (A) or median plane (B); numerical labels identify sound source locations (in degrees). A directionally dependent mid-frequency notch is a prominent feature of the HRTFs. Orientation responses of cat M91cbn6 to bursts of broadband noise (C), $5-18-\mathrm{kHz}$ bandpass noise (D), and $18-\mathrm{kHz}$ highpass noise (E). Lines connect average orientation responses to stimulus locations; ellipses show variability ( \pm 1 S.D.) of responses at each location. The performance deficits that were observed during testing with high-frequency noise suggest that mid-frequency notches are important sound localization cues for cats. Acoustic data taken from Rice et al., 1992; behavioral results are from Huang and May, 1996a.

These observations have led to the hypothesis that auditory processing within the DCN may contribute to directional hearing by decoding HRTF-based spectral cues for sound localization (Young et al., 1992).

A number of previous behavioral studies have failed to demonstrate auditory deficits with DCN lesioning procedures. Masterton and Granger (1988) found that the cat's detection of pure tones or noise bursts did not require an intact dorsal acoustic stria (DAS) or intermediate acoustic stria (IAS). These fiber tracts convey ascending axonal projections from the dorsal and posteroventral cochlear nuclei to the auditory brainstem and midbrain. Hearing sensitivity was severely compromised by lesions of the trapezoid body. Similarly, Masterton et al. (1994) observed that DCN processing was neither necessary nor sufficient for enhancing the detection of auditory signals in the presence of masking noise. Sutherland et al. (1998b) failed to obtain sound localization deficits after DAS/IAS lesions in behavioral tasks that were based on the learned discrimination of sound source elevation.

The first demonstration of a DCN lesioning effect was reported by Sutherland et al. (1998a) who noted a loss of reflexive sound orientation accuracy in the median vertical plane after bilateral DAS/IAS lesions. The reflex orientation paradigm used by the investigators facilitated the behavioral testing of several animal subjects by eliminating the need for long-term training, but a detailed analysis of lesioning effects was not possible in that study because response habituation limited the number of tests that could be repeated within individual subjects. As an alternative behavioral measure, our present study examined the role of the DCN in sound localization behavior using food-reinforced sound orientation and spatial acuity tasks. Our goal was to perform extensive psychophysical testing in highly trained cats before and after the output pathway of the DCN was lesioned with surgical procedures. This psychophysical approach made it possible to extend the orientation measures of Sutherland et al. (1998a) to a more complete range of azimuths and elevations in the frontal sound field. Moreover, the development of a 'learned' head orientation task allowed us to test the hypothesis that DAS/IAS deficits may be less obvious in learned sound localization responses than in reflexive behaviors, as proposed by Sutherland et al. (1998b). Results obtained with these methods indicate that DCN processing is critical for accurate performance in learned orientation tasks just as it is necessary for reflexive orientation; however, the most robust DAS/ IAS deficits in the present study were confined to tasks that demanded absolute identification of sound source locations and not a simple discrimination of directional changes between sound source locations. 


\section{Materials and methods}

\subsection{Subjects}

Experiments were performed on three adult male cats with an extensive history of psychophysical testing. These subjects' orientation responses to bursts of broadband noise were described by May and Huang (1996, see Fig. 1C); their responses to mid-frequency localization cues were reported by Huang and May (1996a, see Fig. 1D,E). Subjects are identified in the text by the first three characters of their identification numbers $\quad(\mathrm{E} 92=\mathrm{E} 92 \mathrm{bm} 6, \quad \mathrm{H} 91=\mathrm{H} 91 \mathrm{az} 4, \quad \mathrm{M} 91=$ M91cbn6). Previous audiometric testing determined that the cats had normal hearing thresholds at frequencies between 6 and $32 \mathrm{kHz}$ (May and Huang, 1996).

The present study tested subjects for approximately 2 years in a sequence of five behavioral paradigms. First, the orientation accuracy of intact cats was assessed under binaural and monaural conditions. Monaural orientation tests were conducted by temporarily inserting a foam ear plug in one ear. Upon completion of pre-lesion baseline measures, the left DAS/IAS was surgically transected. After a 1-month period of postoperative recovery, binaural and monaural orientation tests were repeated for several months until subjects attained their most accurate post-lesion performance. A second surgical procedure was then performed to produce a bilateral DAS/IAS lesion. The post-lesion performance of bilaterally lesioned cats was evaluated with the binaural orientation task as well as a spatial acuity task that measured the discrimination threshold for changes in sound source azimuth and elevation (i.e., the minimum audible angle).

The lesioning procedure was not performed on the right DAS/IAS of cat M91 because this subject experienced major orientation impairments after the unilateral lesion. Subsequent histological evaluation indicated neurological damage spreading well beyond the expected degeneration of the DCN. Results from this subject are presented to distinguish the specific effects of DCN lesions from the more generalized sensorimotor debilitation that may arise as a consequence of manipulations of the lateral brainstem and cerebellum that are necessary during the surgical procedure.

All experimental procedures were approved by the Institutional Animal Care and Use Committee of The Johns Hopkins School of Medicine (NIDCD Grant 5 R01 DC00954: Spectral Cues for Sound Localization). Periodic examinations by veterinary staff ensured that each cat maintained good general health and normal adult weight $(3.5-4.0 \mathrm{~kg})$ during the course of this study.

\subsection{Surgical preparation}

Axons of DCN principal cells (fusiform and giant cells) exit the cochlear nucleus via the DAS and project to the central nucleus of the contralateral inferior colliculus (ICC). As the DAS crosses the dorsal aspect of the restiform body it is joined by the IAS, which is primarily composed of axons originating from octopus cells in the posteroventral cochlear nucleus (PVCN). Our surgical manipulation involves both of these pathways and is referred to as a DAS/IAS lesion (Masterton and Granger, 1988).

The DAS/IAS was lesioned bilaterally in two separate surgical procedures; the left DAS/IAS was lesioned first. Cats were anesthetized with intramuscular injections of xylazine $(0.5 \mathrm{mg} / \mathrm{kg})$ and ketamine $(25 \mathrm{mg} / \mathrm{kg})$, followed by atropine $(0.05 \mathrm{mg} / \mathrm{kg})$ to control mucous secretions. Temporalis muscles were dissected and a $1-\mathrm{cm}$ fenestration was placed in the squamous temporal bone to gain access to the lateral cerebellum. Dura was incised and the cerebellar hemisphere was elevated to expose the projections of the DAS/IAS arising from the dorsal neck of the DCN. The striae form a relatively compact fiber bundle as they pass behind the inferior cerebellar peduncle and were sectioned just above the nucleus by making knifecuts or by aspiration. A period of post-lesion behavioral testing was conducted to determine the effects of a unilateral DAS/IAS lesion, then surgical procedures were repeated in the right brainstem of cats H91 and E92. A bilateral lesion was not attempted in cat M91 because this subject showed almost complete loss of orientation behavior after the unilateral lesion.

\subsection{Histological and electrophysiological assessments of DASIIAS lesions}

The completeness of surgical lesions was assessed with retrograde labeling techniques at the conclusion of post-lesion behavioral testing. The central nucleus of the inferior colliculus (IC) receives direct projections from the contralateral DCN via the DAS (Osen, 1972; Ryugo and Willard, 1985) and from stellate cells in the contralateral ventral cochlear nucleus (VCN) via the trapezoid body (Adams, 1979). The independent pathways linking the dorsal and ventral cochlear nuclei to IC allow incomplete DAS/IAS lesions to be confirmed by the presence of labeled DCN principal cells after injections of retrograde labels are made in the IC. Conversely, the absence of DCN labeling can be interpreted as a complete lesion when the success of the histological procedure is verified by the presence of labeled cells in the VCN. 
Cats were anesthetized with barbiturate (dial urethane) and the ICs exposed by aspirating occipital cortex. Both ICs received multiple injections of wheat germ agglutinin-horseradish peroxidase (WGA-HRP) along the tonotopic (dorsoventral) axis of the nucleus. The surgical exposure was closed and cats were maintained in light anesthesia for $48 \mathrm{~h}$ to allow sufficient time for retrograde transport of label to the cochlear nucleus. Cats were then killed by barbiturate overdose (Nembutal) and immediately perfused. Brainstems were cut into $50-\mu \mathrm{m}$ sections and reacted with tetramethyl benzidine to visualize WGA-HRP labeling using the method of Mesulam (1978).

The pattern of retrograde labeling that was observed in the intact right cochlear nucleus of cat M91 is shown in Fig. 2A. Under low magnification (upper panel), extensive labeling is seen in the fusiform and deep layers of the DCN and throughout the dorsoventral limits of the VCN. Under higher magnification (lower panel), labeled neurons in the DCN can be identified as the more superficial fusiform cells and the deeper giant cells that project axons directly to the central nucleus of the

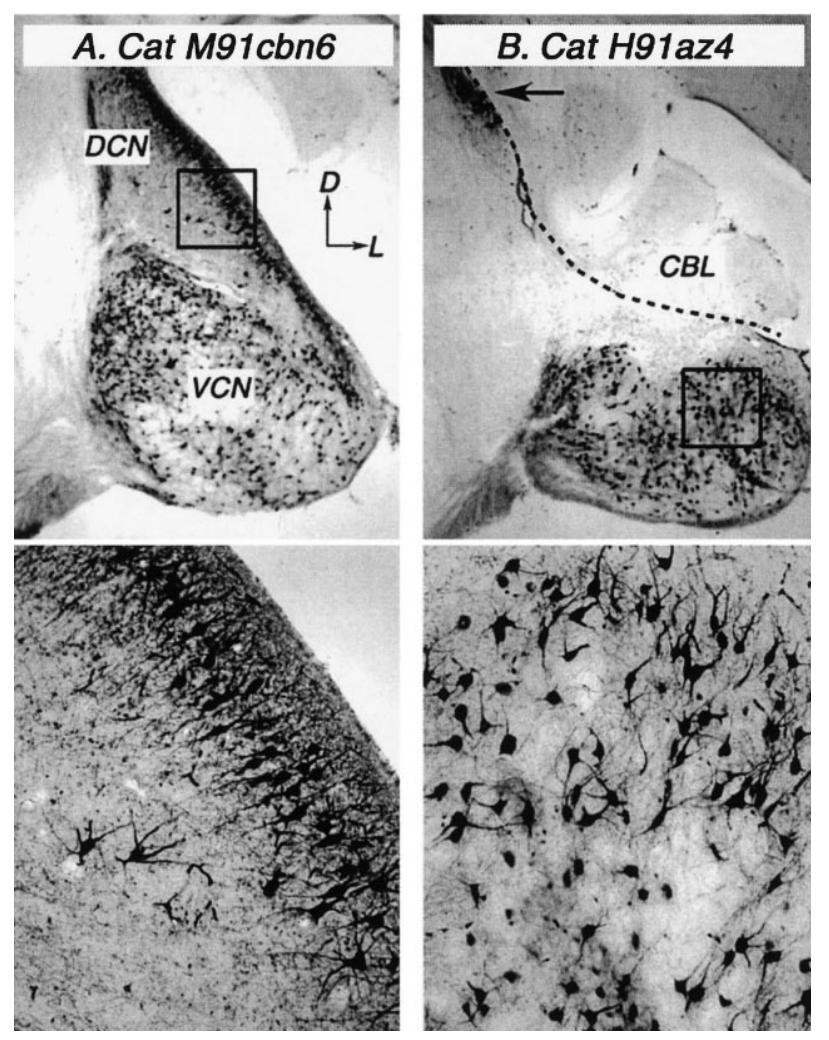

Fig. 2. Retrograde labeling in the intact right cochlear nucleus of cat M91cbn6 (A) and the lesioned right cochlear nucleus of subject H91az4 (B). Labeling within the rectangular fields in the upper panels is shown under higher magnification in the lower panels. CBL, cerebellum; DCN, dorsal cochlear nucleus; VCN, ventral cochlear nucleus; D, dorsal; L, lateral.
IC via the DAS. The pattern of labeling that was associated with a DAS/IAS lesion is shown in Fig. 2B. These histological results were obtained in the right cochlear nucleus of cat H91. As a result of axotomy, principal cells in the medial portions of the DCN have degenerated during the months of behavioral testing that following the surgical procedure. With the loss of tissue, the folium of the cerebellum has collapsed onto the lateral edge of the brainstem. The lesioning procedure did not eliminate all DCN projections to the IC. Clusters of labeled cells were usually observed in the more dorsal portions of the nucleus (arrow in upper panel). The absence of a distinct DCN/VCN boundary makes it difficult to distinguish surviving fusiform cells in the most ventral regions of the DCN from stellate cells in the VCN (lower panel), but the effectiveness of the lesion in the remainder of the nucleus was unmistakable.

Unlike the remaining subjects in the study, the lesioned cochlear nucleus of cat M91 did not show the pattern of retrograde labeling that is described in Fig. 2B. Instead, this subject exhibited widespread tissue loss throughout the lateral brainstem. Visual inspections during histological preparation suggested that the subject's most severe pathology bordered the site of the DAS/IAS lesion and involved the cochlear nucleus, vestibular nucleus, and lateral cerebellum. A more detailed light microscopic confirmation of these initial impressions was not possible because this highly degenerated material failed to withstand the sectioning procedure.

Electrophysiological tests of hearing sensitivity were performed on cats $\mathrm{H} 91$ and E92 immediately before the surgical preparation for WGA-HRP labeling. The thresholds of just detectable click-evoked auditory brainstem responses (ABRs) for both ears of these bilaterally lesioned subjects fell within the range of normal variability for unlesioned controls in our laboratory. ABR testing was not performed on cat M91.

\subsection{Acoustic stimuli}

Gaussian noise bursts were synthesized using an array processor (Tucker Davis Technologies AP2) and digital-to-analog converter (Tucker Davis Technologies DD1) with a sampling rate of $100 \mathrm{kHz}$. Stimuli were gated with an electronic switch (Tucker Davis Technologies SW2) using $\cos ^{2}$-shaped rise/fall times of $10 \mathrm{~ms}$. The average spectrum level of the noise bursts was $5 \mathrm{~dB}$ SPL. A programmable attenuator (Tucker Davis Technologies PA4) randomized the overall level of individual noise bursts using a uniform distribution of $\pm 5 \mathrm{~dB}$ (increments of $1 \mathrm{~dB}$ ) to eliminate loudness cues that may have coincided with speaker changes. Signals were presented from high-frequency speakers with closely matched frequency response characteristics (Ra- 
dio Shack, Super Tweeter). All speakers showed a relatively flat output at frequencies between 5 and $20 \mathrm{kHz}$ that deviated by less than $\pm 3 \mathrm{~dB}$ across speakers. Representative calibrations are shown in Fig. 3. A fresh noise burst was synthesized for each stimulus presentation. The magnitude spectrum of each stimulus was divided into $100-\mathrm{Hz}$ bins and then scrambled using a uniform distribution of $0-8 \mathrm{~dB}$ to minimize the importance of potential cues derived from any remaining differences in speaker response characteristics (Wightman and Kistler, 1989).

\subsection{Behavioral assessments of DAS/IAS lesions}

The cats' ability to identify the free-field location of auditory stimuli was measured with a food-reinforced sound orientation task (May and Huang, 1996). Unlike more traditional reflex-based procedures, this paradigm was able to sample thousands of behavioral responses from each subject across a variety of experimental conditions. At the conclusion of orientation testing, the cats' discrimination thresholds for changes in sound source azimuth and elevation were evaluated with a food-reinforced spatial acuity task (Huang and May, 1996b).

\subsubsection{The sound orientation task}

The sound orientation task has been used in our previous behavioral studies of directional hearing in cats (May and Huang, 1996; Huang and May, 1996a), and was described in greater detail by May and Huang (1996). The task was based on three trial conditions. Fixation trials ensured that each testing sequence started with cats placing their head in a standardized orientation of $0^{\circ}$ azimuth (AZ), $0^{\circ}$ elevation (EL). Fixed-orientation trials trained cats to follow fixation with a head pointing response that was directed toward the location of a brief noise burst. For these tests, the subject was required to meet a criterion of accuracy to within $10^{\circ}$ of the sound source location and to hold the response for a few seconds while head position was measured with a search coil device (Polhemus Isotrak; Colchester, VT). The search coil creates an electromagnetic field around the subject and tracks the three-dimensional movements of the head by inducing currents in a head-mounted sensor. This system is more fully described in May and Huang (1996). Free-orientation trials were identical to fixed-orientation trials except that no accuracy criterion was in effect. In this trial context, the cat's head movements were assumed to reflect the perceived location of the sound source rather than procedural rules.

Fixation trials started when the cat pressed down on a lever near its right front paw. The observing response produced a single $40-\mathrm{ms}$ noise burst from the fixation

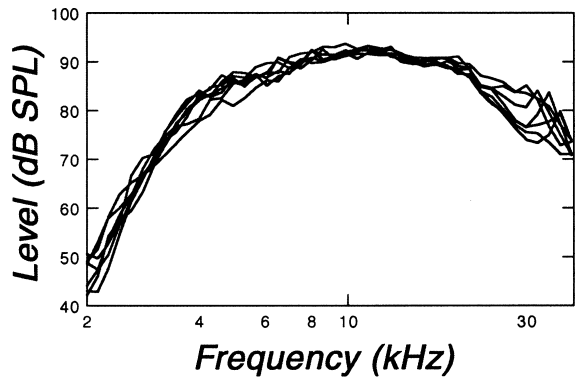

Fig. 3. Acoustic calibrations of the free-field speaker array. These representative functions were obtained from seven different speakers at randomly selected locations in the frontal sound field.

speaker at $0^{\circ} \mathrm{AZ}, 0^{\circ} \mathrm{EL}$. The cat was required to hold down on the lever while directing its head toward the fixation speaker. At the conclusion of a 3-5-s silent interval following the initial noise burst, head position was checked to confirm that the subject was oriented to within $\pm 10^{\circ}$ of the fixation speaker. If the alignment criterion was met, an LED on the fixation speaker flashed on for $40 \mathrm{~ms}$ and the cat obtained a food reward by releasing the lever within 2 s. For trials in which the head was not properly positioned, the LED flash was delayed until the cat made a correct fixation response. Fixation trials were reset to their initial values if the cat released the lever at any time other than the 2 -s response window that went into effect at the onset of the LED flash. Experiments were performed in a dimly lit chamber to minimize visual cues regarding speaker placements and to enhance the perceptual impact of LED flashes.

Fixed-orientation trials were added to the testing sequence once cats accurately performed fixation trials. As in fixation trials, fixed-orientation trials started with a noise burst from the fixation speaker; however, the LED on the fixation speaker did not flash after the criterion for fixation was achieved. Instead, a second noise burst was delivered from one of eight randomly selected speaker locations. These locations changed on a daily basis. The cat was required to redirect its head from the fixation point to within $\pm 10^{\circ}$ of the orientation speaker. Head position was checked after a 3-5-s silent interval and an accurate orientation response produced an LED flash from the orientation speaker; whereupon the cat obtained a food reward by releasing the lever within $2 \mathrm{~s}$. If the orientation response failed to meet the criterion for accuracy, the trial was terminated without the LED flash. Fixed-orientation trials were designed to enforce orientation accuracy by only accepting head movements that were properly directed toward specific sound source locations.

Free-orientation trials were introduced at the end of training to provide a less controlled context for sampling orientation behavior. For these trials, the LED 
flashed on the orientation speaker regardless of the subject's head position. Results are based only on responses that were measured during free-orientation trials. Trials were selected at random with the following probabilities: fixation trials, 0.15 ; fixed-orientation trials, 0.20; and free-orientation trials, 0.65.

Methods for summarizing orientation responses are shown in Fig. 4 using pre-lesion data from cat E92. Data collected during one session are presented in Fig. 4A,B. The eight randomly selected speaker locations for this session are indicated in Fig. 4A. Responses are plotted in double-pole coordinates so that directional changes (described in degrees $\mathrm{AZ}$ and $\mathrm{EL}$ ) are of constant arc length for both central and eccentric locations on the spherical speaker array, as discussed by Middlebrooks et al. (1989). Lines in Fig. 4A follow head trajectories during a representative trial to each location. Initial movements are completed within $1 \mathrm{~s}$ after the onset of the noise burst and the head is held in a stable position for $2-4 \mathrm{~s}$ while the cat waits for the LED on the orientation speaker to flash. Fig. 4B shows the average azimuth and elevation of stable head orientation for all trials in the session. The tight clustering of responses around target locations indicates that intact cats make accurate and consistent orientation responses when tested with broadband noise bursts (Huang and May, 1996a).

The accuracy of orientation responses was assessed for several months prior to the DCN lesions. During this period, the position of the eight test speakers changed daily with any location within the speaker array potentially available for testing. Speaker locations above $75^{\circ} \mathrm{EL}$ or below $-45^{\circ} \mathrm{EL}$ were not tested because of physical limitations in the speaker array. Summary orientation measures were derived by averaging the data from different speaker locations using spatial bins that spanned the frontal field with a resolution of $30^{\circ} \mathrm{AZ}$ and EL. The double-pole coordinates of bin locations are indicated by the intersection of grids in Fig. 4.

Lines in Fig. 4C connect the grand averages of multisession orientation responses to the locations of the orientation speakers. Ellipses indicate the variability of responses that contributed to the grand averages. The $x$-dimension of each ellipse represents \pm 1 S.D. of the range of response azimuths for the spatial bin (i.e., the range of right-left errors); the $y$-dimension equals \pm 1 S.D. of response elevations (up-down errors). As shown in Fig. 4C, summary data for intact cats indicate small errors and little variability for orientation responses to the more centrally located speaker positions. The underestimation of more lateral or highly elevated speaker locations by E92 is a common response pattern that is assumed to result from a decrease in the quality of spectral cues at more eccentric sound locations (May and Huang, 1996; Sutherland et al., 1998a).

After the conclusion of pre-lesion orientation testing, the importance of binaural directional cues was evaluated by testing cats with one ear occluded by a foam ear plug (Cabot Safety; Southbridge, MA). Subjects were habituated to the ear plug by having them wear the device during feeding or exercise periods in the animal quarters. Once subjects were accustomed to temporary monauralization, the ear plug was inserted a few minutes before the start of behavioral sessions and removed at the end of the sessions. The plug was lubricated with a topical anesthetic (Xylocaine, 2.5\% lidocaine) to facilitate placement and to improve the seal of the ear canal. The effectiveness of the plugging procedure was evaluated at the conclusion of behavioral testing by measuring thresholds of click-evoked ABRs in both ears of cats H91 and E92, first with the ear open and then with the ear plug in place, the average threshold shift was $29 \mathrm{~dB}$.
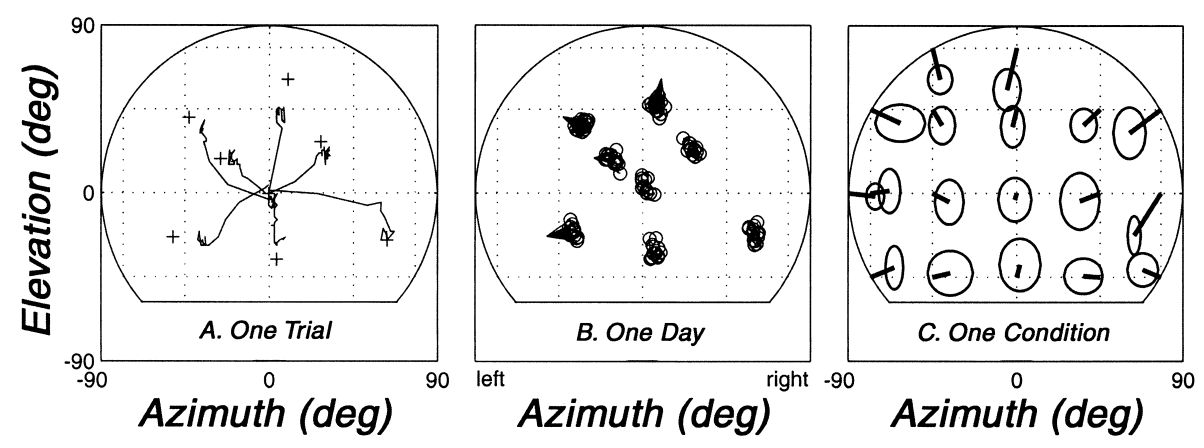

Fig. 4. Pre-lesion orientation behaviors of cat E92bm6. A: Head trajectories toward the source location of a single burst of broadband noise for each of eight randomly selected speaker locations (plus symbols). B: Final position of all orientation responses in one session (9/13/96); lines connect responses to speaker locations. C: Complete sample of broadband orientation responses for cat E92bm6 (3258 trials). Data are binned at intervals of $30^{\circ} \mathrm{AZ}$ and EL. Lines connect grand average responses to the center of spatial bins; ellipses are centered on average responses and show spatial distribution of responses within each bin ( \pm 1 S.D.). 


\subsubsection{Spatial acuity task}

The spatial acuity task is a modification of the minimum audible angle procedure that was described in detail by Huang and May (1996b). In the current study, this paradigm was used to demonstrate that cats were able to discriminate changes in sound source location even after their sound orientation behaviors were disrupted by DAS/IAS lesions.

Spatial acuity trials were initiated when the cat pushed down on the response lever with its right front paw. As the cat held down on the lever, 20-40 noise bursts ( $250 \mathrm{~ms}$ in duration) were presented at a rate of 2 bursts/s from a speaker located at $0^{\circ} \mathrm{AZ}, 0^{\circ} \mathrm{EL}$. These standard stimuli were followed by a 2 -s interval in which the sound source alternated between the standard speaker and a comparison speaker at some other location. If the cat released the lever during the period of speaker alternations (hit), $1 \mathrm{ml}$ of liquefied meat paste was delivered to the subject's mouth. Releasing the lever before the speaker alternations or failing to release the lever during the alternations was punished with a 5-s time out during which no acoustic stimuli were presented.

Catch trials were presented on $20 \%$ of all trials to estimate each cat's tendency to release the lever in the absence of the sound localization cues that were associated with speaker changes. The catch trial procedure involved prolonging presentations of stimuli from the standard speaker for an additional 2-s interval, which was the duration of speaker alternations in a spatial acuity trial. If the subject correctly held through the period of additional standard stimulus presentations, a comparison trial was presented and cats could then obtain a food reward by releasing the lever during speaker alternations. Releases during catch trials (false alarms) were followed by time out intervals.

Psychometric functions were created by sampling hit and false alarm rates at three positive azimuths in the interaural horizontal plane (to the right of the cat at $6^{\circ}$, $12^{\circ}$, and $18^{\circ} \mathrm{AZ}$ ) or at three positive elevations in the median vertical plane $\left(6^{\circ}, 15^{\circ}\right.$, and $30^{\circ}$ EL). Speaker locations were selected to produce near threshold discrimination when sounds were presented from the comparison speaker in closest proximity to the standard speaker, and near perfect performance at the largest angular separation. Physical dimensions of the test speakers (Realistic Super Tweeters) allowed a minimum speaker separation of $6^{\circ}$.

\section{Results}

Orientation results from each cat are based on more than 10000 trials that were sampled during periods when the subjects showed stable optimum performance after experimental manipulations. Pre-lesion binaural and monaural trials were obtained during a 7-month period that preceded surgical transection of the left DAS/IAS. After the surgical procedure, subjects were given a 1-month hiatus from behavioral testing. The effects of unilateral lesions were then studied for 4 months. When the evaluation of unilaterally lesioned cats was completed, a second surgical lesion was made in the right DAS/IAS of cats E92 and H91. Both subjects were able to return to post-operative retraining sessions after 1 week of recovery. Once post-operative performance stabilized, the effects of bilateral lesions on orientation behavior were studied daily for 2 months. Cat M91 continued to produce unilateral lesion data throughout this period without exhibiting any indication of improved performance. During the final months of the study, all three cats were trained to perform the spatial acuity task. Minimum audible angles reflect data that were combined over 1 month of testing.

\subsection{Effects of DCN lesions on sound orientation}

Effects of DCN lesions on sound orientation behavior are shown in Fig. 5. Table 1 summarizes the number of sessions and trials, and the average error and variation scores for cats E92 and H91. All three cats showed relatively accurate performance during pre-lesion baseline testing (Fig. 5A), although there was a general tendency to underestimate the location of the orientation speaker at the highest elevations and most lateralized azimuths. This 'undershoot' has been attributed to a diminution of mid-frequency spectral cues at eccentric sound locations (Rice et al., 1992; May and Huang, 1996). Underestimation of negative elevations is not apparent in these data because the design of the speaker array did not allow testing at elevations below $-45^{\circ}$.

Cats showed increases in orientation error and response variability after unilateral lesion of the left DAS/IAS (Fig. 5B). In two of the three cats (E92 and H91) the most substantial loss of performance was confined to higher elevations ipsilateral to the lesion. Smaller deficits were noted at other locations in the frontal field, while near normal performance was seen at the more front and center test locations. The more generalized neural degeneration in cat M91 resulted in a complete loss of orientation accuracy. Although capable of making large inaccurate head movements, this cat typically maintained a fixation response (head pointed toward $0^{\circ} \mathrm{AZ}, 0^{\circ} \mathrm{EL}$ ) regardless of the location of the orientation speaker.

Further decreases in orientation accuracy were observed after a second surgical procedure was performed to lesion the right DAS/IAS of cats E92 and H91 (Fig. 5C). Both bilaterally lesioned subjects showed increases in average response error and variability relative to pre- 


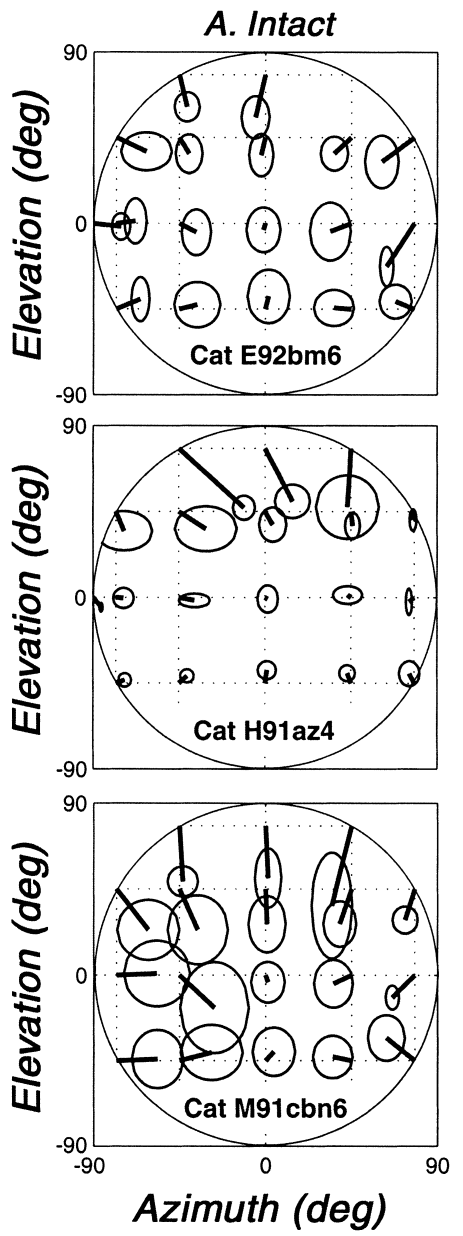

B. Unilateral (left)
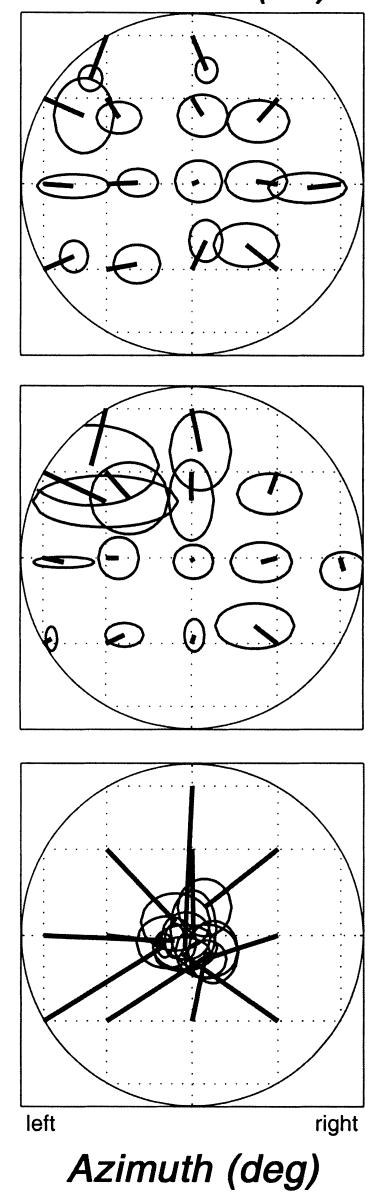

C. Bilateral
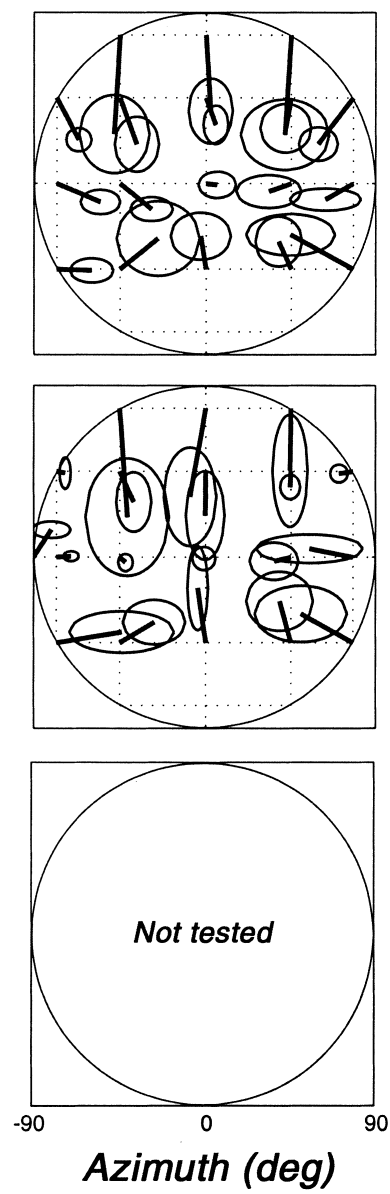

Fig. 5. Performance of three cats in the sound orientation task prior to DAS/IAS lesions (A), after a unilateral lesion of the left striae (B), and after a second lesion was made in the right striae (C). Cat M91az4 showed complete loss of orientation accuracy after the left DCN was lesioned and did not receive a lesion of the right DCN.

lesion performance; however, at most locations, losses of elevation accuracy tended to be larger in magnitude than azimuthal deficits.

Non-parametric sign tests (Armitage and Berry, 1987) were used to evaluate the statistical significance of lesioning effects. For example, to determine the significance of the increase in elevation error shown by cat E92 after the bilateral lesion, error difference scores $\left(x_{i}\right)$ were calculated by subtracting the average post-lesion error in each spatial bin (i) from pre-lesion errors.

Table 1

Summary statistics for effects of DAS/IAS lesions on orientation behavior

\begin{tabular}{|c|c|c|c|c|c|c|}
\hline Cat & Sessions & Trials & AZ error & EL error & AZ S.D. & EL S.D. \\
\hline \multicolumn{7}{|c|}{ Pre-lesion } \\
\hline E92bm6 & 31 & 3258 & 8.9 & 7.9 & 8.1 & 10.9 \\
\hline $\begin{array}{l}\text { H91az4 } \\
\text { Left D }\end{array}$ & 33 & 3094 & 5.9 & 8.5 & 6.6 & 6.7 \\
\hline E92bm6 & 32 & 2283 & $12.0^{* *}$ & 8.4 & $13.0^{*}$ & 9.8 \\
\hline \multicolumn{7}{|c|}{ Bilateral DAS/IAS lesion } \\
\hline E92bm6 & 15 & 1272 & 11.4 & $19.8^{*}$ & $13.8^{* *}$ & 11.0 \\
\hline H91az4 & 21 & 1990 & 9.2 & $15.4^{* *}$ & $12.3^{*}$ & $13.4^{*}$ \\
\hline
\end{tabular}

Values indicate total number of sessions and trials for each testing condition and average error or S.D. scores for all spatial bins tested under different conditions. Statistical significance is based on differences in pre-lesion versus post-lesion error and S.D. scores for the same spatial bins $\left({ }^{*} P<0.05 ;{ }^{* *} P<0.01\right)$. 

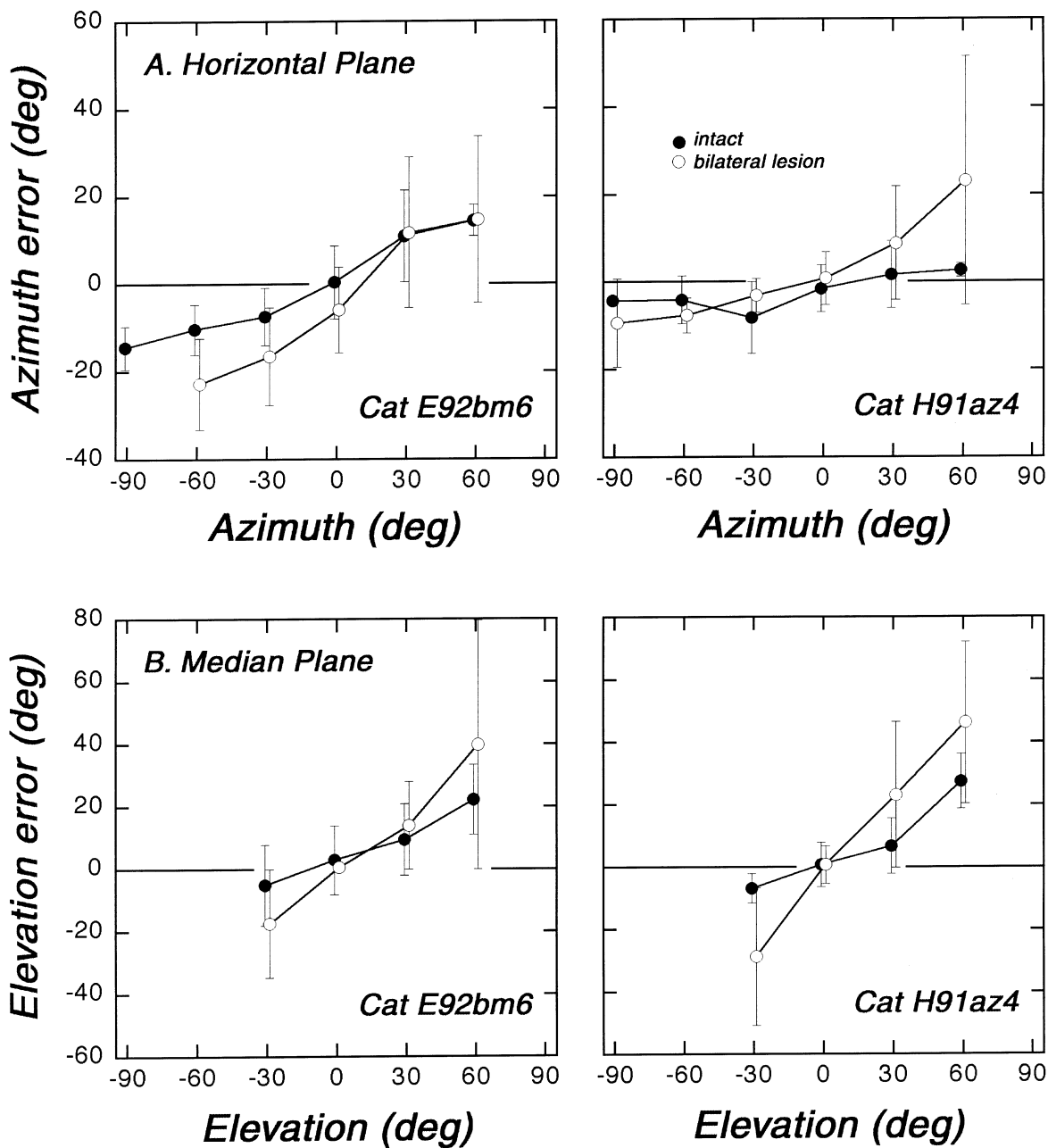

Fig. 6. Orientation accuracy of cats E92bm6 and H91az4 for sound sources in the interaural horizontal plane (A) and median vertical plane (B). Filled symbols plot performance prior to surgical manipulations; open symbols indicate performance after the completion of bilateral DAS/IAS lesions. Error bars show the S.D. of responses in each spatial bin.

Under the assumption that the bilateral lesion had no effect on orientation behavior, positive and negative values of $x_{i}$ have equal likelihood and should follow a binomial distribution. Instead, the bilateral lesion data of cat E92 yielded negative $x_{i}$ values (i.e., larger elevation errors after the lesion) for 13 out of 17 spatial bins. This departure from chance expectancies was statistically significant $(P<0.05)$. Cat $\mathrm{H} 91$ also showed significant increases in elevation error after the bilateral lesion (16 out of 19 bins, $P<0.005$ ). The sign test is a conservative method in this application because statistical significance is based on the number of spatial bins showing deficits and not on the magnitude of loss in individual bins. Although the S.D.s of cat H91 almost doubled after the bilateral lesion relative to pre-operative values, the increases were confined to five out of 19 bins and therefore did not attain statistical significance in the sign test. Each test condition showing statistically significant lesioning effects is identified with asterisks in Table 1.
Fig. 6 provides a direct comparison of the orientation behaviors of cats E92 and H91 before and after bilateral DAS/IAS lesions. Here, results are limited to azimuth errors in the interaural horizontal plane (Fig. 6A) and elevation errors in the median vertical plane (Fig. 6B). Although both cats showed increased errors for many sound source locations in the horizontal plane after the lesioning procedure, these deficits were restricted to sound source azimuths in either the left (E92) or right hemisphere (H91). Locations in the opposite sound field elicited near normal performance. This clustering of accurate orientation responses contributed to the lack of statistical significance for azimuth measures in bilaterally lesioned cats (Table 1). By contrast, surgical transections of DAS/IAS projections increased response errors at both positive and negative source elevations in the median plane. These lesioning effects were statistically significant in both cats (Table 1). 


\subsection{Effects of DCN lesions on spatial acuity}

Cats were tested with the spatial acuity task to confirm that the effects of DAS/IAS lesions on orientation accuracy did not reflect general hearing loss, lack of motivation, or inability to perform the operant task; the results of these tests are shown in Fig. 7. All subjects produced very high detection rates for changes in speaker location when the standard and comparison speakers were arranged in the horizontal plane (Fig. 7A), even when tested with the smallest speaker change available in the apparatus $\left(6^{\circ} \mathrm{AZ}\right)$. Cats showed false alarm rates (releases during catch trial presentations) that averaged less than $20 \%$. A signal detection analysis of these data yielded a discrimination threshold (the smallest change in azimuth producing $d^{\prime}=1$ ) of less than $6^{\circ}$, which corresponds well with previously published minimum audible angles for normal cats (Martin and Webster, 1987; Heffner and Heffner, 1988; Huang and May, 1996b).

It could be argued that normal spatial acuity in the horizontal plane is expected for cats with bilateral DAS/IAS lesions because discrimination of changes in azimuth may be primarily determined by binaural processing of interaural time differences (ITDs) and interaural level differences (ILDs) that do not require the
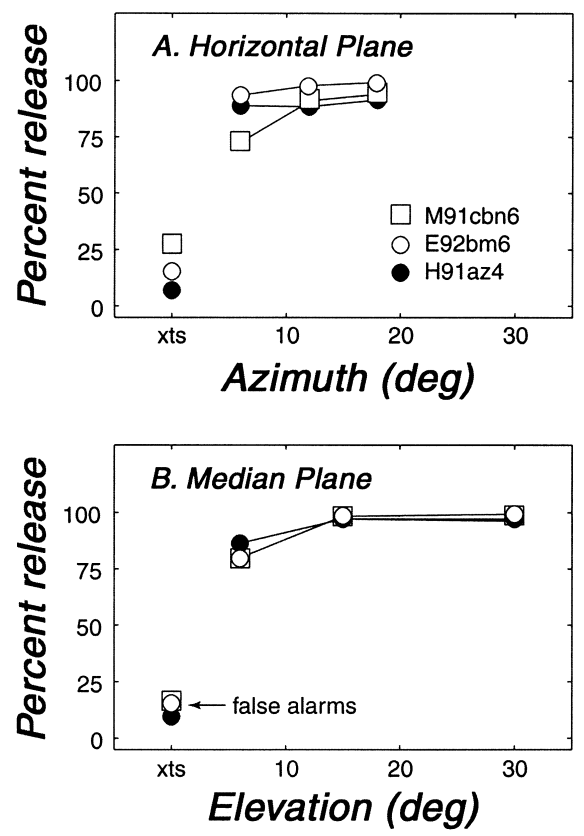

Fig. 7. Effects of DCN lesions on spatial acuity. These tests were performed after bilateral lesions in cats E92bm6 and H91az4, and after a unilateral lesion in cat M91cbn6. Psychometric functions plot the percentage of trials in which the subject correctly released a response lever during a period of alternations between two speaker locations as a function of the magnitude of the directional changes. Results are shown for tests in the horizontal (A) and median plane (B). No change in location occurred during catch trials (xts).
DCN. In fact, this interpretation may explain why response azimuth was less affected than response elevation during orientation tests with bilaterally lesioned cats (Fig. 6). To minimize the importance of interaural cues, spatial acuity was also measured in the median plane (Fig. 7B). The pattern of discrimination for changes in elevation was essentially the same as results obtained in the horizontal plane. Detection rates remained above $75 \%$ for all comparison speaker locations, while false alarms rates were less than $20 \%$. Again, these data predict minimum audible angles that fall within the range of normal performance.

\subsection{Effects of monaural ear plugging on sound orientation}

Effects of ear plugging on the accuracy of sound orientation may explain the absence of large behavioral deficits after unilateral DAS/IAS lesions. These results are shown in Fig. 8 using data from cat H91. Orientation responses in Fig. 8A were obtained prior to the lesion with the right ear plugged. Although increases in error and variability were noted under monaural testing conditions, the cat continued to make reasonably well directed head movements toward speaker locations ipsilateral to the open ear. This pattern of accurate behavior persisted even at the more frontal locations on the side of the plugged ear. If it is assumed that the plugging procedure modified pinna filtering effects in the plugged ear and binaural directional cues, it is likely that the monauralized cat performed the task by attending to HRTF-based spectral cues in the unoccluded ear. Based on these results, it is not surprising that cats with unilateral DAS/IAS lesions also performed the orientation task well (Fig. 5B) since these subjects could rely on binaural time and level difference cues as well as monaural spectral cues in the intact ear.

An alternative explanation for the conservation of accurate orientation behavior in monauralized cats is that the ear plugging procedure may have failed to substantially disrupt binaural cues for sound localization. In particular, low-frequency interaural delay cues can persist even with substantial sound attenuation in the plugged ear (Wightman and Kistler, 1997). Given the electrophysiologically measured attenuation of the plug and the steep low-frequency roll-off of the speaker system (Fig. 3), noise levels at $2 \mathrm{kHz}$ were approximately $80 \mathrm{~dB}$ less than levels at $10 \mathrm{kHz}$, and therefore should have been well below detection thresholds in this frequency region.

An additional test of the importance of binaural localization in ear-plugged cats was evaluated by repeating monaural tests after bilateral DCN lesions. As shown in Fig. 8B, a substantial loss of performance was observed when the plug was placed in the right 

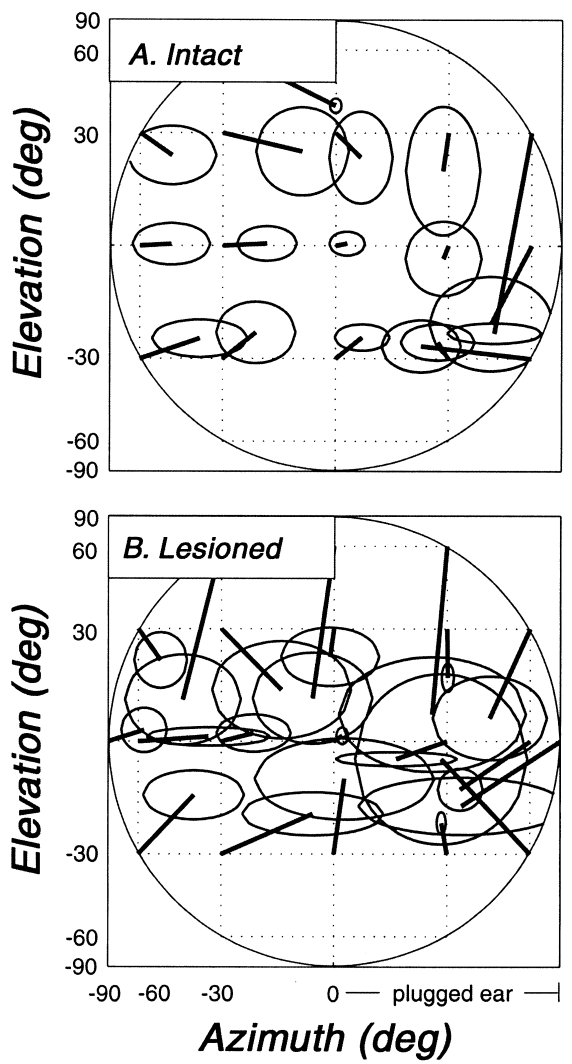

Fig. 8. Monaural orientation responses of cat H91az4 (right ear plugged). Results are shown before (A) and after bilateral DCN lesions (B).

ear of the bilaterally lesioned cat H91. Since the major pathways carrying ITD and ILD information from the VCN to the superior olivary complex (SOC) were not compromised by the surgical procedure, a response based on interaural delay cues would be expected to produce performance that more closely matched the results shown in Fig. 8A. Instead, these differences in the subject's pre-lesion versus post-lesion performance suggest that DCN processing of spectral cues is necessary to achieve accurate orientation to sound source elevation and may be sufficient for accurate orientation to sound source azimuth when binaural cues are unavailable.

\section{Discussion}

The main finding of this study is that cats with DAS/ IAS lesions show a loss of performance in a sound orientation task. In two of three subjects, a bilateral lesion was required to produce large elevation errors at most spatial locations in the frontal sound field. By contrast, lesioned cats showed spatial acuity for sound sources in the horizontal and median planes that was equivalent to the minimum audible angles of unlesioned cats in our previous studies (Huang and May, 1996b) and those of other investigators (Casseday and Neff, 1975; Martin and Webster, 1987; Heffner and Heffner, 1988). The following discussion evaluates the specificity of the functional deficits that follow DAS/IAS lesions, the relative importance of binaural versus spectral information in spatial hearing tasks, and the putative physiological basis of DAS/IAS lesioning effects.

\subsection{Is the loss of orientation accuracy following DAS/IAS lesions a DCN deficit?}

Spectral integration properties of DCN principal cells (Spirou and Young, 1991; Nelken and Young, 1994) have led to the proposal that the DCN may be involved in the auditory processing of spectral cues for sound localization (Young et al., 1992). That hypothesis is supported by our observation that DAS/IAS lesions produced behavioral deficits in the sound orientation task. Although a loss of DCN processing is likely to have contributed to these results, it cannot be stated unequivocally that damage to the DCN is the only source of orientation deficits in the present study because lesioning procedures also damaged IAS projections carrying information in and out of the PVCN.

Most ascending IAS projections are octopus cell axons that terminate in the ventral nucleus of the lateral lemniscus (VNLL: Warr, 1969; Adams and Warr, 1976). The thick diameter and calyceal endings of octopus cell fibers suggest a pathway that is capable of rapidly conducting cochlear nucleus activity to the VNLL. In addition, single-unit recordings from octopus cells show a precisely timed response at stimulus onset (Godfrey et al., 1975; Rhode et al., 1983; Rouiller and Ryugo, 1984). These anatomical and physiological specializations have implicated the PVCN as a component of the acoustic startle reflex (Davis et al., 1982). Potential consequences of a disruption of PVCN processing on sound orientation behavior are unknown since the HRTF sensitivity of PVCN onset units has not been established. Nor is it known how binaural processing in ventral auditory pathways may be modified by the loss of descending projections that enter the cochlear nucleus by way of the DAS/IAS (Adams, 1976). Nevertheless, relatively normal binaural hearing was suggested by each cat's performance in the spatial acuity task.

The DCN lies on the dorsolateral surface of the medulla beneath the paraflocculus of the cerebellum and immediately caudal to the inferior cerebellar peduncle. These surrounding structures can be injured during the surgical retraction to expose the DCN or during lesioning procedures if knifecuts are not confined to the target structure. The potential for cerebellar damage is of particular concern in a study of orientation behavior 
because the ensuing loss of balance or motor control can compromise a cat's ability to make coordinated head movements; when this impairment occurs, subjects may perceive normally but perform poorly.

Cats H91 and E92 showed no signs of vestibular or motor deficits after bilateral DAS/IAS lesions. These subjects interacted with other cats during exercise periods and were able to execute $1-\mathrm{m}$ vertical leaps without unusual effort as they entered and exited their home cage. Informal testing with the head tracking device indicated that the cats could rotate their heads well beyond $\pm 90^{\circ} \mathrm{AZ}$ and $\pm 70^{\circ}$ EL to obtain food rewards. Cat M91 displayed a similar range of head movements but vestibular and cerebellar damage was suggested by the broad-based gait that this subject assumed when walking to and from the testing apparatus. Subsequent histological evaluation of the brainstem of this subject confirmed extensive tissue damage including the lateral cerebellum and vestibular nuclei. Unlike the other two subjects in this study, cat M91 showed large orientation errors after the unilateral lesion presumably because of more generalized sensorimotor deficits. Although collateral damage to neighboring structures of the DCN confounded interpretations of the effects of DAS/IAS lesions in cat M91, the post-operative orientation behaviors of this subject illustrate a different pattern of behavioral deficit for nonspecific lesions.

In conjunction with ABR measures in cats $\mathrm{H} 91$ and E92, spatial acuity testing in all three cats suggested normal hearing sensitivity and good discrimination of directional changes after DAS/IAS projections were severed. Previous DAS/IAS lesioning studies by Masterton and colleagues also failed to note significant changes in detection thresholds (Masterton and Granger, 1988; Masterton et al., 1994) and spatial discrimination (Sutherland et al., 1998b). ABRs were not obtained from cat M91, but it is unlikely that normal auditory function was maintained in the left ear of this subject given the extensive neural degeneration of the lesioned cochlear nucleus and ventral acoustic stria (Masterton and Granger, 1988).

\subsection{Are separate processes involved in the identification of azimuth and elevation?}

Cats with DAS/IAS lesions exhibited their largest behavioral deficits when orienting to stimulus elevation. This observation is consistent with the interpretation that spectral cues processed in the DCN are critical for determining the elevation of sound sources. Localization of stimulus azimuth, on the other hand, may rely on ILDs or ITDs that are primarily conveyed to binaural neurons in the SOC via ventral pathways that are not directly affected by DAS/IAS lesions. A directional bias in the importance of spectral versus binaural information is supported by psychoacoustic studies in human listeners. If HRTF filtering effects are confounded by narrowing the bandwidth of free-field stimuli, human subjects make accurate assessments of sound source azimuth but large errors in the identification of stimulus elevation (Middlebrooks, 1992). These errors suggest a processing scheme in which stimulus elevation is derived from spectral cues by finding the best match between the frequency of a narrowband stimulus and the peaks in the HRTF filter function at different sound source locations. By contrast, binaural processes appear to play the primary role in the determination of stimulus azimuth. Wightman and Kistler (1992) have shown that binaural time difference cues dominate the perception of azimuth for low-frequency stimuli when an unnatural conflict is created in the ITD and ILD directional signatures of virtual sound sources (i.e., closedfield sounds that simulate free-field directional properties).

Results obtained with human subjects indicate that localization of stimulus azimuth is based on redundant binaural and spectral information, and that listeners may attend to whatever cues are most reliable in a given testing situation. Similar contextual effects are seen in studies with cats. For example, cats showed substantial errors in response azimuth and elevation when orientation responses were probed with high-pass filtered noise bursts (Fig. 1E) or mid-frequency pure tones (Huang and May, 1996a). Thus, performance was adversely affected by removing mid-frequency spectral cues and low-frequency binaural cues. A convenient method for distinguishing the relative importance of these two forms of directional information is to 'monauralize' the subject by placing a plug in one ear. While the effectiveness of ear plugging procedures for achieving a natural monaural state is debatable (Wightman and Kistler, 1997), erroneous ITD and ILD cues that result from attenuating high-frequency sounds in one ear would be expected to alter localization performance. In our study, temporarily monauralized cats maintained good orientation accuracy in the hemifield of the unplugged ear (Fig. 8A), but only prior to DAS/IAS lesions (Fig. 8B). By contrast, when auditory processing of spectral information was compromised by damaging the DCN, cats lost the ability to direct the head toward sound source elevation but continued to make reasonably accurate responses to stimulus azimuth (Fig. 5C). It can be concluded from these results, just as it was in studies of human listeners, that spectral cues are necessary for localization of stimulus elevation by cats, whereas binaural and spectral cues alone or in combination are sufficient for the determination of stimulus azimuth.

Cats with DAS/IAS lesions exhibited large elevation errors when tested with the orientation task (Fig. 6B), 
but performed the spatial acuity task as well as normal controls (Fig. 7). This finding suggests that fundamental differences may exist between the auditory processes governing the absolute identification of sound source locations and the relative spatial discrimination of changes between locations. Sutherland and colleagues noted similar task-specific deficits in their studies of DAS/IAS lesioning effects. Because their analysis was based on reflexive head orientation measures that demonstrated behavioral deficits (Sutherland et al., 1998a) and an operantly conditioned elevation discrimination task that did not (Sutherland et al., 1998b), these investigators proposed that DAS/IAS lesioning effects may be most apparent in unlearned reflexive auditory behaviors. Our present study offers a direct test of this prediction by evaluating the effects of DAS/IAS on behavioral performance in a learned head orientation task. The observation that both learned and reflexive head orientation responses are compromised by DAS/IAS lesions suggests that the actual distinction between the two procedural contexts may be due to differences in the auditory processes that contribute to accurate identification of sound source locations versus the precise discrimination between sound sources.

Our studies of the auditory nerve representation of spectral cues for sound localization in cats support the existence of these dual processes (May and Huang, 1997). Movements of a sound source in the horizontal or median planes lead to broadly distributed changes in auditory nerve fiber discharge rates. The largest rate changes in the population response are seen among neurons that are tuned to high frequencies where the HRTF exerts the most robust directionally dependent filtering effects on the magnitude spectrum of a complex sound. Consistent with the interpretation that this rate information represents a neural code for the discrimination of directional changes, cats perform well in spatial acuity tasks when tested with high-frequency noise bursts (Huang and May, 1996b). The unique directional characteristics of mid-frequency spectral notches appear to dominate the absolute identification of sound source locations. The significance of these spectral cues has already been demonstrated in behavioral studies of cats (Huang and May, 1996a), and is summarized in Fig. 1. Given the critical importance of accurately locating sound sources, it is not surprising that the central auditory pathways have evolved specialized processes for extracting narrowband spectral features from their auditory nerve inputs (Young et al., 1992). Our current behavioral study suggests a functional link between these biologically relevant sounds and the unusual spectral integration properties of the principal output neurons of the dorsal cochlear nucleus (Spirou and Young, 1991; Nelken and Young, 1994).

\section{Acknowledgements}

P. Stiles, A. Krause, and Jennifer Whiteside provided animal care and conducted behavioral tests. J. Rice measured head-related transfer functions shown in Fig. 1; A. Huang performed noise bandwidth manipulations described in Fig. 1. This research was supported by the National Institute on Deafness and Other Communication Disorders (5 R01 DC00954).

\section{References}

Adams, J.C., 1976. Single unit studies on the dorsal and intermediate acoustic striae. J. Comp. Neurol. 170, 97-106.

Adams, J.C., 1979. Ascending projections to the inferior colliculus. J. Comp. Neurol. 18, 519-538.

Adams, J.C., Warr, W.B., 1976. Origins of the cat's acoustic strias determined by injection of horseradish peroxidase into severed tracts. J. Comp. Neurol. 170, 107-122.

Armitage, P., Berry, G., 1987. Statistical Methods in Medical Research. Blackwell Scientific, Oxford, pp. 409-410.

Casseday, J.H., Neff, W.D., 1975. Auditory localization: Role of auditory pathways in brain stem of the cat. J. Neurophysiol. 38, 842-858.

Davis, M., Gendelman, D.S., Tischler, M.N., Gendelman, P.M., 1982. A primary acoustic startle circuit-lesion and stimulation studies. J. Neurosci. 2, 791-805.

Godfrey, D.A., Kiang, N.Y.S., Norris, B.E., 1975. Single unit activity in the posteroventral cochlear nucleus of the cat. J. Comp. Neurol. $162,247-268$.

Heffner, R.S., Heffner, H.E., 1988. Sound localization acuity in the cat: Effects of azimuth, signal duration, and test procedure. Hear. Res. 36, 221-232.

Huang, A.Y., May, B.J., 1996a. Sound orientation behavior in cats: II. Mid-frequency spectral cues for sound localization. J. Acoust. Soc. Am. 100, 1070-1080.

Huang, A.Y., May, B.J., 1996b. Spectral cues for sound localization in cats: Effects of frequency domain on minimum audible angles in the median and horizontal planes. J. Acoust. Soc. Am. 100, 2341-2348.

Martin, R.L., Webster, W.R., 1987. The auditory spatial acuity of the domestic cat in the interaural horizontal and median vertical planes. Hear. Res. 30, 239-252.

Masterton, R.B., Granger, E.M., 1988. Role of the acoustic striae in hearing: Contribution of dorsal and intermediate acoustic striae to detection of noise and tones. J. Neurophysiol. 60, 1841-1860.

Masterton, R.B., Granger, E.M., Glendenning, K.K., 1994. Role of acoustic striae in hearing: Mechanism for enhancement of sound detection in cats. Hear. Res. 73, 209-222.

May, B.J., Huang, A.Y., 1996. Sound orientation behavior in cats: I. Localization of broadband noise. J. Acoust. Soc. Am. 100, 10591069.

May, B.J., Huang, A.Y., 1997. Spectral cues for sound localization in cats: A model for discharge rate representations in the auditory nerve. J. Acoust. Soc. Am. 101, 2705-2719.

Mesulam, M.M., 1978. Tetramethyl benzidine for horseradish peroxidase neurohistochemistry: a non-carcinogenic blue reaction product with superior sensitivity for visualizing neural afferents and efferents. J. Histochem. Cytochem. 26, 106-117.

Middlebrooks, J.C., 1992. Narrow-band sound localization related to external ear acoustics. J. Acoust. Soc. Am. 92, 2607-2624. 
Middlebrooks, J.C., Makous, J.C., Green, D.M., 1989. Directional sensitivity of sound-pressure levels in the human ear canal. J. Acoust. Soc. Am. 86, 89-108.

Nelken, I., Young, E.D., 1994. Two separate inhibitory mechanisms shape the responses of dorsal cochlear nucleus type IV units to narrowband and wideband stimuli. J. Neurophysiol. 71, 24462462.

Osen, K.K., 1972. Projection of the cochlear nuclei on the inferior colliculus in the cat. J. Comp. Neurol. 144, 355-372.

Rhode, W.S., Oertel, D., Smith, P.H., 1983. Physiological response properties of cells labelled intracellularly with horseradish peroxidase in cat ventral cochlear nucleus. J. Comp. Neurol. 213, 426447.

Rice, J.J., May, B.J., Spirou, G.A., Young, E.D., 1992. Pinna-based spectral cues for sound localization in cat. Hear. Res. 58, 132-152.

Rouiller, E.M., Ryugo, D.K., 1984. Intracellular marking of physiologically characterized cells in the ventral cochlear nucleus of the cat. J. Comp. Neurol. 225, 167-186.

Ryugo, D.K., Willard, F.H., 1985. The dorsal cochlear nucleus of the mouse: A light microscopic analysis of neurons that project to the inferior colliculus. J. Comp. Neurol. 242, 381-396.

Spirou, G.A., Young, E.D., 1991. Organization of dorsal cochlear nucleus type IV unit response maps and their relationship to activation by bandlimited noise. J. Neurophysiol. 66, 1750-1768.
Spirou, G.A., May, B.J., Wright, D.D., Ryugo, D.K., 1993. Frequency organization of the dorsal cochlear nucleus in cats. J. Comp. Neurol. 329, 36-52.

Sutherland, D.P., Masterton, R.B., Glendenning, K.K., 1998a. Role of acoustic striae in hearing: Reflexive responses to elevated sound sources. Behav. Brain Res. 97, 1-12.

Sutherland, D.P., Glendenning, K.K., Masterton, R.B., 1998b. Role of acoustic striae in hearing: Discrimination of sound-source elevation. Hear. Res. 120, 86-108.

Warr, W.B., 1969. Fiber degeneration following lesions in the posteroventral cochlear nucleus of the cat. Exp. Neurol. 23, 140-155.

Wightman, F.L., Kistler, D.J., 1989. Headphone simulation of freefield listening. I: Stimulus synthesis. J. Acoust. Soc. Am. 85, 858867.

Wightman, F.L., Kistler, D.J., 1992. The dominant role of low-frequency interaural time differences in sound localization. J. Acoust. Soc. Am. 91, 1648-1661.

Wightman, F.L., Kistler, D.J., 1997. Monaural sound localization revisited. J. Acoust. Soc. Am. 101, 1050-1063.

Young, E.D., Spirou, G.A., Rice, J.J., Voigt, H.F., 1992. Neural organization and responses to complex stimuli in the dorsal cochlear nucleus. Phil. Trans. R. Soc. Lond. B Biol. Sci. 336, 407-413. 